\title{
Pitfalls in Healthcare Delivery!! Time to Switch to Collaborative Care
}

\author{
Vinuth Chikkamath, Vaishnavi Naik, Sucharitha and Anantha Naik Nagappa* \\ Department of Pharmacy Practice, SCS College of Pharmacy, India
}

Submission: February 01, 2018; Published: February 05, 2018

*Corresponding author: Anantha Naik Nagappa, SCS College of Pharmacy, Harapanahalli-583131, Karnataka, India, Email: anantha1232000@gmail.com

\section{Opinion}

The medical practice in India is handled by the private practitioners who run the outpatient department clinics (OPD) of general medicine and specialty clinics. These OPD clinics are obstructing change over to the collaborative care model. In collaborative care models Doctors, Nurses, and Pharmacists work on a patient to provide the safe, efficacious and cost effective treatments.

Drugs and Diagnostics are important in Management of diseases. Drugs and some diagnostics need to be administered into the body for therapeutic and diagnostic purposes. These are administered to the body are considered as Xenobiotics/foreign substances in the facto are handled by metabolism and excretion mechanisms of the system. If the molecule is fat soluble, it is rendered water soluble by various biochemical reactions. So that the drug can be thrown out by the excretion. The Xenobiotics which are administered to the body for therapeutic purposes sometimes can cause serious damage and causality. The Adverse Drug Reactions of the drugs is usually dose dependent and symptoms of ADR manifests as the dose increase exceeding the minimum toxic concentration. Recently it has been so happening the Carbamazepine adult dose is administered to a child by mistake resulting in toxicity of the Carbamazepine leading to death. The doctor in charge is questioned regarding the cause of death by the patient relatives. It is a very common scenario when medicine is practiced by independent stand alone Clinicians. Such types of medical practice by independent, clinician are who is not having any supporting healthcare professionals as Pharmacist and Nurses. Such Clinicians acclaim popularity in community by manipulating the patient condition by the use of steroids or some other therapeutic agents which give instant relief from the symptoms. The patient believes the Doctor is really intelligent who can reverse the symptoms and morbidity of the patient instantaneously [1].

Here an independent practitioner who runs a clinic based treatment never dares to face the public wrath in times of calamity. If we ponder a bit it is evident that it would be very simple if we change the style of medical practice from independent module to the patient-centric care. The patientרcentric care is the concept of the World Health Organization (WHO) which emphasizes on the welfare of the patient by a team of health workers. This concept also described as equity in health care wherein the Doctors, Nurses and Pharmacists, along with the inputs from the patients make the foundation of the treatment. To excel in the patient centric care we must understand the importance contributions of each stake holders. The team of Doctors, Nurses, and Clinical Pharmacists work in a collaborative manner to bring the best outcomes which are safe and effective. We cannot blame an individual if the serious ADRs have happened in the patient due to lack of inputs as in stand-alone waste clinical intervention. The popular and convenient stand alone clinic is under severe criticism as unable to save the life of the patient, which was either not so serious in routine practice. Currently, we are seeing the Doctors, becoming extremely busy and rapidly prescribing patients after patients in order to serve all the patients to the waiting. In this process the Doctors are unable to diagnose properly and usually end up with prescribing the symptomatic treatments along with empirical prescriptions. Due to lack of systematic approach of medical care it is just a on spot prescribing aimed at the management of the patient condition in terms of fever, cough, and cold. Thus the patient visiting the clinic end up with poor outcomes mandating the patients to get treated in corporate hospitals or teaching hospitals likely to fall ill again and again and seeing the same Doctor $[2,3]$.

The principles of health care delivery should be scientific and based on evidence rather than mitigating and the science and symptoms of the disease. The systematic approach of the health care delivery should be rational and safe. However, to switch over from the existing health care delivery system to modernized healthcare delivery. The current module of self regulation should be switched into explainable and accountable health care delivery. The system may also insist upon sharing the treatment details to the patient and provider. They also update the treatment continued like in highly regulated health care systems of the US and Canada. There should be a public 
domain wherein the documentation of treatments provided is uploaded for the benefits of the patient and government bodies. The documents of treatment given should be accessible to all stake holders who can view and criticize the line of therapy so that the patients and fellow health care workers are able to view and understand the intension of treatment. The secrecy of treatment given is an old passion approach which is having the burden of risk of ADRs of the drug and aristocratic approach by the prescriber is likely to become corrupt and collaborate with pharmaceutical industry helping them to market the products at the cost of patients' health $[4,5]$.

\section{Conclusion}

The concept of collaborative care if the Pharmacist and Nurse is going to cause the revolution in health care delivery wherein the patients get the fare deal for the money they spent for their health. It can put a break to the irrational use of medicines it is also may help the Pharmacist and Nurses to contribute for the patient wellness and cost-effective treatment. Hence, there should be a national debit on How to switch over from the product-centric prescribing to patient-centric treatments in which the goals of safety, efficacy and cost-effectiveness are embedded. In the current style of treatments where the Doctor alone is sole responsible for safety, efficacy and cost of the treatment.

\section{References}

1. Calderón JL, Beltrán RA (2004) Pitfalls in Health Communication: Healthcare Policy, Institution, Structure, \& Process. Med Gen Med 6(1): 9 .

2. https://www.cdc.gov/nchs/data/isp/035_health_data_issues_for_ primary_health_care_delivery_system_in_develp_countries.pdf

3. http://www.gih.org/files/FileDownloads/Transforming\%20 Health\%20Care\%20Delivery\%20Primer\%20March\%202012.pdf.

4. https://www.nap.edu/read/10548/chapter/7

5. https://endingthedocumentgame.gov/PDFs/HealthcareDelivery.pdf 